\title{
Multimedia Pre-Lab Training and Testing
}

\author{
Bruno Korst, P.Eng. \\ Manager, Undergraduate Hardware Labs \\ The Edward S. Rogers, Sr. Department \\ of Electrical and Computer Engineering \\ University of Toronto \\ bkf@comm.utoronto.ca
}

\begin{abstract}
In most courses involving laboratory practices, students must dedicate some time to prepare for experiments. Usually, this preparation is done in writing, on paper or on a lab book, and is submitted to staff for scrutiny before an experiment. In some cases, students are required to endure further preparation if they are found to be lacking in the knowledge of critical or dangerous procedures. This paper presents a pre-lab training tool which encompasses the presentation of relevant material formatted in multiple media, as well as the creation of tests to prepare and gauge student retention. The tool is intended to assist both students and lab personnel alike, by allowing quick and easy addition of both teaching material and questions for pre-lab evaluations.
\end{abstract}

\section{Introduction}

Lab experiments require preparation from those taking it, as well as from those delivering it. For instructors and staff, it is wise to ensure all students have undergone some preparation prior to experiments, particularly those experiments involving critical routines. Traditionally, pre-lab assignments are given to students in print, and students either read a doc- ument, or answer a number of questions and hand them over to the personnel in charge of running the experiment. For those labs involving meticulous routines, dangerous or potentially harmful machinery, circuitry or substances, students are usually subject to a live, in-lab presentation or quiz. Oftentimes, however, students are observed "preparing" in the ten minutes preceding the experiment.

Understandably, the traditional practice usually consumes a significant amount of lab time that could be used for actual experimentation. Indeed, the time used for the instruction of very specific safety procedures must be allocated, but it can be transferred out of the curriculum lab time if one can ensure that the students have seen and pondered about the key scenarios prior to entering the lab [1]. The experiment can then be conducted after a brief review of the procedures and student assessment rather than a full review and instruction of every scenario.

This paper proposes an alternative methodology for laboratory preparation. A webbased tool is designed to allow students access to chronological posts, documents, simulation files, audio and video. Most importantly, it allows for testing the students by using the material in all these formats, and assessing their re- 
tention prior to coming to the lab practice. This tool also allows instructors and lab personnel to add content quickly to the course page (in video, for instance) in order to generate new material for instructing and testing as new scenarios emerge during experiments in the lab. All content material can be used to create a database of questions, which in turn is used to generate a fixed number of random questions to test every student who logs in. Instructors and lab personnel are able to keep track of the students who logged in and took the test, as well as their marks. If the mark reaches an acceptable level indicating good retention of the material, the student is allowed to perform the experiment. Otherwise, the student may be asked to revisit the material and take the test as needed until the material is learned. The webbased tool described below has applications in the delivery of practical tutorials, preparation for lab experiments as well as safety training, or any kind of training involving practical scenarios which demand assessment of student retention. Different applications (a microcontroller programming tutorial as opposed to a safety preparation on lathe operation or on the mixing of volatile substances) will have different demands in retention from the students.

\section{Using a New Visual Format}

While paper-based or text-based lab preparation material has been used successfuly through the years, the use of video has become more prevalent in practical training. The newer generation of students seems to absorb more readily information that is presented in a shortened length, more condensed in content and that makes use of a variety of stimuli. A plain, 20 minute-long video clip with one visible announcer on white background is likely to be ignored if compared to a 5 minute-long one with plenty of camera movement, a variety of extra noises and voice, but no announcer in sight. This is to say that, arguably, the mate- rial to be added to a lab preparation tool would work better with new students if it follows to some degree the very familiar format of entertainment environments such as YouTube or of very popular video games. The need to ellicit attention by adjusting the delivery format to fit the audience is not in itself a novelty [2]. The novelty is the creation of a specific training and testing tool allowing for all different media formats to be presented.

The tool to be described in the next section uses a web-log ("blog") style posting where video or audio clips can be embedded in the posts, if possible in a short, objective and very upfront manner. In addition to the posts, tests can be created also as part of the preparation, giving instructors the option to require a minimum level of retention prior to experiments. The ease to add material in the form of scenarios in video allows for a very dynamic instruction, as students from one group can be prepared and tested (in safety, for instance) based on actual scenarios which occurred to previous groups.

\subsection{A Multimedia Web-Based Tool}

A web-based tool was designed [3] to allow for this novel lab preparation format. A snapshot of this tool is presented in figure 1 . There are two administrative levels: one to create new courses and assign administrators to the courses and a second one to manage students, content and assessment (tests). Courses may have more than one administrator assigned, so that both the professor and the lab instructor can add content to the course page. This, of course, requires good communication between the individual course administrators, as tests will be created based on the content added.

Student assessment is done by generating random tests to students who log in. The questions to these tests are selected at random from a database of questions, which may include material in plain text (ascii), in pictures or in 


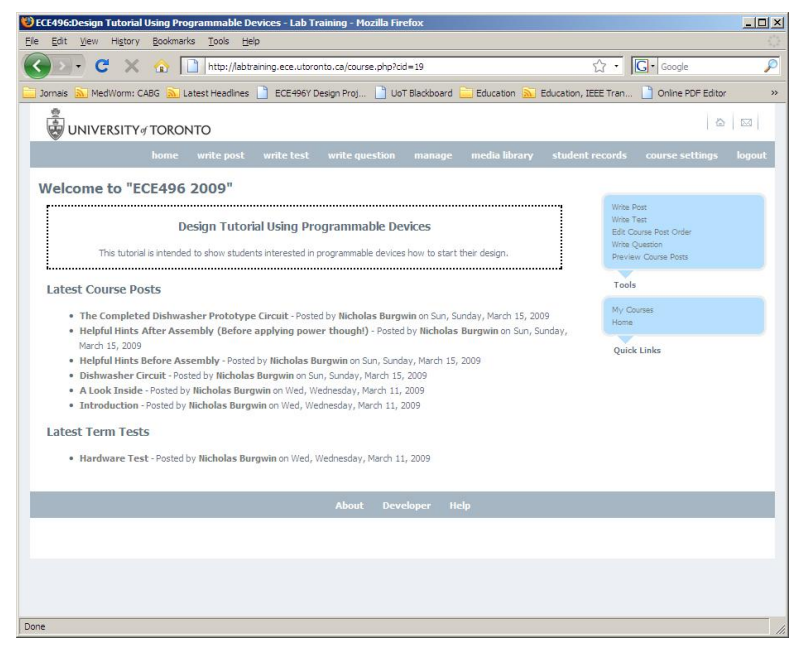

Figure 1: Labtraining Web Tool

video. The length of the tests is up to the instructor, and the instructor should be careful in monitoring the questions database, so that the length of the tests does not compromise the intention to create individual, random, tests.

\subsection{Supporting Hardware}

In addition to a web server with large memory and bandwidth capacity to support short video streams, lab personnel should be equipped with small, easy to use video cameras. Recently, cheap flash-based video cameras have become popular following the new generation's desire to post videos about nearly everything around them. Such cameras are cheap (i.e., less than US\$200) and USB-ready, many without the need for an external cable. Three cameras were evaluated during the development of this tool: two Flip cameras [4] and an Insignia camera [5]. Among those evaluated, the Flip Video Mino has proven to be the most accessible and easy to use. Its greatest advantages are the one-button operation and the built-in USB connector, while its greatest disadvantate is the rather reduced screen size. The software needed for the downloading of the video clips comes in the camera. No disc or download is needed. The Insignia camera had a fold-out screen (larger than the Flip cameras) and a thicker body, which made its handling a little slower. It also needed an external USB cable.

During the development of the tool as well as the initial content, the emphasis is on the simplicity of operation. The camera is a tool that the lab personnel must have ready to catch potential teaching scenarios as they happen. In this regard, the Flip camera proved to be the best option.

\subsection{New Scenarios and New Questions}

It is very common for instructors conducting lab experiments to observe students threading the wrong path towards resolving a particular problem in the experiment. Though it is important to let students learn by their mistakes, one can use these real scenarios to show other students what not to do, and to test them if necessary. The lab training web tool allows for the quick addition of new scenarios in video by uploading a recorded clip to a video library, which can in turn be used to generate both an entry in the material presented and a test question. Since it is advisable for the lab instructor to have a portable camera, the lab instructor can generate an entry and a question for the next lab group almost immediately after he observes a situation that should be brought to everyone's attention.

\subsection{Other Uses}

This tool was originally designed to provide support to electrical engineering laboratories. However, it is by no means limited to this area and may very well be used in other engineering fields, chemistry, biology, medicine, dentistry, as well as in the instruction of a variety of technical trades.

In addition to lab preparation and testing, this tool can also be utilized in tutorials and short-term courses. One instance is the instruction of all-practical "put it together" sessions 
for microcontroller-based systems. This tool is presently being used for a short course on designing a mini-washing machine, which encompasses the following topics:

- designing a microcontroller-based system and creating a bill of materials (BOM);

- programming the microcontroller and debugging the code;

- laying-out the printed-circuit board and fabricating it;

- populating the board and debugging the hardware;

As it can be easily seen, the tool will assist the integration of multiple disciplines with text, schematics and video, for the completion of the project.

\section{Conclusion}

A good lab preparation is the one which promotes and facilitates the retention of the material contained in the related experiment. A lengthy lab preparation is not necessarily better than a short one in a more naturally familiar format. This paper presented a webbased tool which allows for the use of multiple types of media in presenting technical material to students preparing for experiments and taking tutorials. It has also presented thoughts on the formatting of such material, as newer generations of students in all areas become naturally acquainted with newly evolving media formats.

\section{Acknowledgments}

The author would like to thank Mr. Raymond Lo and Mr. Nicholas Burgwin, for their valuable contribution to the development of the tool itself and for the initial material. Also, the author is very thankful for the funding received from the Office of the Provost, University of Toronto.

\section{References}

[1] M. Abdulwahed and Z. K. Nagy, "Towards Constructivist Laboratory Education: Case Study for Process Control Laboratory", Frontiers in Education Conference, Saratoga Springs, 2008.

[2] N. Postman, "Amusing Ourselves to Death: Public Discourse in the Age of Show Business", 1st ed. Penguin, USA, 1985.

[3] http://labtraining.ece.utoronto.ca

[4] Flip Video Cameras, http://ca.theflip.com

[5] Insignia High Definition 5MP digital camcorder, http://insigniaproducts.com 